\title{
The impact of body composition on skeletal muscle strength in Caucasian women: implications for sarcopenia screening
}

\author{
K. Shilland and H. D. McCarthy \\ Public Health Nutrition Research Group, London Metropolitan University, Holloway Road, London N7 8DB, UK
}

Sarcopenia is rapidly gaining significance as a public health concern within today's ageing population. It is currently defined as an age-related loss of muscle mass, combined with loss of strength, functionality, or both ${ }^{(1)}$. The associated co-morbidities, in particular, the adverse coexistence of sarcopenia and metabolic syndrome highlight the need to identify effective screening tools and prevention strategies $^{(2)}$. Since skeletal muscle and adipose tissue have opposing effects on glucose metabolism, the ratio of skeletal muscle to fat may be of particular significance for health outcomes. However, this muscle-fat balance is not currently incorporated into the working definition of sarcopenia. This study therefore sought to identify the influence of body composition within the etiology of the condition.

Study participants comprised of 34 Caucasian women aged between 35 and 45 years (BMI range $17 \cdot 5-41 \cdot 7 \mathrm{~kg} / \mathrm{m}^{2}$ ), recruited from an affluent area in South East London. Height, weight and waist circumference (WC) were measured and fat mass (FM, kg), \% body fat, fat-free mass (FFM, kg) and appendicular skeletal muscle mass (SMMa, kg) were estimated using segmental, single frequency bioelectrical impedance analysis. SMMa was expressed as a percentage of total body mass (\% SMMa) and muscle-to-fat ratio (MFR) was calculated. Muscle strength was determined by handgrip dynamometry and expressed as a ratio to body mass (GS: Mass). Spearman's RHO correlations identified relationships between body composition indicators and GS:Mass ratio.

GS:Mass was not significantly related to SMMa $(\mathrm{kg})(\mathrm{r}=-0 \cdot 1, \mathrm{p}>0 \cdot 05)$ however, significant positive relationships were observed between GS:Mass and \% SMMa $(r=0.73, p<0.01)$ and GS:Mass and MFR $(r=0.81, p<0.01)$. Significant inverse relationships were observed between GS:Mass and \% body fat $(\mathrm{r}=-0.75, \mathrm{p}<0.01)$ and between GS:Mass and WC $(\mathrm{r}=-0.64, \mathrm{p}<0.01)$.

These findings suggest that body fat may be an important predictor of sarcopenia risk due to its negative impact on skeletal muscle strength. Such findings therefore suggest a potential need to reevaluate sarcopenia definitions to also consider the impact of body composition in the etiology of the condition, particularly given the overwhelming prevalence of overweight and obesity in today's ageing population. In addition, this study further highlights the value of WC measurements as a potential assessment tool for sarcopenia. Furthermore, it is suggested that research in this area could be supported by the implementation of a GS:Mass ratio calculation which may have the potential to better predict absolute strength than GS alone. However, further research is needed to verify this suggestion.

1. Cruz-Jentoft AJ, Baeyens JP, Bauer JM et al. (2010) Age Ageing 39, 412-423.

2. Atlantis E, Martin SA, Haren MT et al. (2009) Metabolism. 58, 1013-22. 